\title{
De novo genome assembly and analysis unveil biosynthetic and metabolic potentials of Pseudomonas fragi A13BB
}

\author{
Opeyemi K. Awolope ${ }^{1}$ (D) Noelle H. O'Driscoll ${ }^{1}$ (D), Alberto Di Salvo ${ }^{1}$ (D) and Andrew J. Lamb ${ }^{2^{*}}$ (D)
}

\begin{abstract}
Objectives: The role of rhizosphere microbiome in supporting plant growth under biotic stress is well documented. Rhizobacteria ward off phytopathogens through various mechanisms including antibiosis. We sought to recover novel antibiotic-producing bacterial strains from soil samples collected from the rhizosphere. Pseudomonas fragi A13BB was recovered as part of this effort, and the whole genome was sequenced to facilitate mining for potential antibiotic-encoding biosynthetic gene clusters.

Data description: Here, we report the complete genome sequence of $P$. fragi A13BB obtained from de novo assembly of Illumina MiSeq and GridION reads. The $4.94 \mathrm{Mb}$ genome consists of a single chromosome with a GC content of 59.40\%. Genomic features include 4410 CDSs, 102 RNAs, 3 CRISPR arrays, 3 prophage regions, and 37 predicted genomic islands. Two $\beta$-lactone biosynthetic gene clusters were identified; besides, metabolic products of these are known to show antibiotic and/or anticancer properties. A siderophore biosynthetic gene cluster was also identified even though $P$. fragi is considered a non-siderophore producing pseudomonad. Other gene clusters of broad interest identified include those associated with bioremediation, biocontrol, plant growth promotion, or environmental adaptation. This dataset unveils various un-/underexplored metabolic or biosynthetic potential of $P$. fragi and provides insight into molecular mechanisms underpinning these attributes.
\end{abstract}

Keywords: Pseudomonas fragi, $\beta$-Lactone antibiotics, Plant growth-promoting rhizobacteria, Rhizosphere microbiome

\section{Objective}

The rhizosphere has been described as one of the most complex ecosystems on Earth, harboring abundant dynamic plant-microbe and microbe-microbe interactions. Plant growth-promoting rhizobacteria (PGPR) are one of the components of this ecosystem where they promote plant growth by enhancing uptake of nutrients and inorganic elements, or by increasing resistance to various environmental stresses including heavy metals, high salt concentrations and phytopathogens $[1,2]$. PGPR protect

\footnotetext{
* Correspondence: a.lamb@rgu.ac.uk

${ }^{2}$ Graduate School, Robert Gordon University, The Ishbel Gordon Building, Garthdee Road, Aberdeen AB10 7QE, Scotland

Full list of author information is available at the end of the article
}

against phytopathogens through a variety of mechanisms, including the ability to gain competitive advantage for nutrients and trace elements and/or produce one or more antibiotics effective against such pathogens $[1,2]$. Whilst the latter characteristic (which is common to many soil dwelling bacteria) has been exploited to develop many clinically useful antibiotics, it remains the case that less than $1 \%$ of all known bacterial species have had their metabolic capabilities exploited in this way [3]. We therefore sought to recover potential novel antibiotic-producing bacterial strains from soil samples collected from the rhizosphere of various plants. Pseudomonas fragi strain $\mathrm{A} 13 \mathrm{BB}$ was isolated as part of this effort. 
P. fragi is a Gram-negative, rod-shaped, aerobic psychrophile. It is widely distributed in nature and commonly associated with meat and dairy spoilage $[4,5]$. It is rarely reported as a PGPR except by Selvakumar et al [5] and Fahr et al [6] who reported its phosphate solubilisation activity and its ability to improve tolerance against aluminium stress in acidic soils, respectively. However, to the best of our knowledge, it has not been previously reported as an antibiotic producer. Therefore, being a species not readily associated with antibiotic production, the genome of $P$. fragi $\mathrm{A} 13 \mathrm{BB}$ was sequenced to facilitate mining for potential antibioticencoding secondary metabolite biosynthetic gene clusters (smBGCs) and other gene clusters that may be responsible for its environmental adaptation and plant growth promotion.

\section{Data description}

P. fragi A13BB was isolated from the rhizosphere of a plant in Aberdeen, Scotland (57.101 N 2.078 W) using an ultra-minimal substrate medium (data file 1) [7]. Purified strain was cultivated in nutrient broth (Oxoid, UK) at $28^{\circ} \mathrm{C}$ for $24 \mathrm{~h}$ before gDNA was extracted from pellets with the DNeasy ${ }^{\circ}$ Ultraclean $^{\circ}$ Microbial Kit for DNA Isolation (Qiagen, UK). The extract was used as template to amplify the $16 \mathrm{~S}$ rRNA gene in PCR reactions using $27 \mathrm{~F}$ and U1510R universal primers, with thermocycler parameters set as follows: Initial denaturation at $95^{\circ} \mathrm{C}$ for 2 min followed by 30 cycles of further denaturation at $95^{\circ} \mathrm{C}$ for $30 \mathrm{~s}$, primer annealing at $45^{\circ} \mathrm{C}$ for $30 \mathrm{~s}$ and elongation at $72{ }^{\circ} \mathrm{C}$ for $105 \mathrm{~s}$. A final elongation was carried out at $70^{\circ} \mathrm{C}$ for $5 \mathrm{~min}$. Amplified DNA fragment was sequenced using the $27 \mathrm{~F}$ primer. Isolate was subsequently identified by $16 \mathrm{~S}$ rRNA gene comparison as $P$. fragi with $99 \%$ identity score.

Libraries were prepared for Illumina sequencing by Glasgow Polyomics (Glasgow, UK) using the Nextera XT DNA Library Preparation Kit (Illumina, USA) following manufacturer's protocol, and sequenced with the Illumina MiSeq using a $300 \mathrm{bp}$ paired end protocol. Libraries were prepared for GridION sequencing by MicrobesNG (Birmingham, UK) using the Oxford nanopore SQK-RBK004 kit and/or SQK-LSK109 kit with Native Barcoding EXP-NBD104/114 (ONT, UK), and sequenced on a FLO-MIN106 (R.9.4 or R.9.4.1) flow cell in a GridION (ONT, UK).

Illumina reads were trimmed with Trimmomatic [8] v0.36 operated in the sliding window mode with Q25 quality cut-off and minimum read length of 100 . The quality of trimmed reads was assessed with FastQC [9] v0.11.8 and results were aggregated with MultiQC [10] v1.8 (data file 2) [11]. Mean quality score across each base position was $\geq 31$. Quality assessment of GridION reads was performed with NanoPlot [12] v1.28.2. Quality statistics are summarised in data file 3 [13], while average read quality plot is displayed in data file 4 [14].

Paired short reads and long reads were assembled de novo with Unicycler [15] v0.4.8. Assembly quality was assessed with Quast [16] v5.0.2. Two contigs were identified (data file 5) [17], the smaller contig (5386 bp) representing the complete genome of bacteriophage $\phi X 174$ (control spike in Illumina sequencing) was subsequently extracted from the data. The larger contig (4, $940,458 \mathrm{bp}$ ) represents the complete genome of $P$. fragi A13BB with sequencing depths of 226x and 32x for Illumina and GridION sequencing, respectively. Assembly completeness was $99.2 \%$ as assessed with BUSCO [18] v4.1.2 using the pseudomanadales_odb10 lineage dataset (data file 6) [19]. Assembly graph was visualised with Bandage [20] and displayed in data file 7 [21]. ANI analysis with the FastANI tool [22] v1.3 confirmed identity as $P$. fragi with the ANI value of 98.9071. Gene and functional annotations were performed with PGAP [23] v4.13 and RASTtk [24] v2.0. Metabolic pathway analyses were performed using the KEGG database [25] Rel 93.0. CRISPRs were identified by CRISPRCasFinder [26], genomic islands were predicted by IslandViewer 4 [27], prophages were identified by PHASTER [28] and smBGCs were identified with antiSMASH [29] v5.1.2. All bioinformatics tools used for genome assembly and analyses were operated with default parameters or as specified in the text.

The complete genome of $P$. fragi A13BB comprises a single chromosome $4,940,458$ bp in size with a GC content of $59.40 \%$. Genomic features include 4410 CDSs, 25 rRNA, 73 tRNA, 4 ncRNA, 3 CRISPRs, 3 prophage regions and 37 predicted genomic islands (data file 8) [30]. Also, 353 subsystems comprising of various gene clusters including those associated with bioremediation, environmental adaptation, biocontrol, and plant growth promotion were identified (data file 9) [31]. Two $\beta$-lactone smBGCs, both showing low homology (20\%) to known smBGCs, were identified. $\beta$-lactones are known for their antibiotic, anticancer and antiobesity properties [32]. A siderophore smBGC was identified even though $P$. fragi is considered a non-siderophore producing member of the genus Pseudomonas [33]. Arylpolyene and NAGGN smBGCs were also identified which, along with the siderophore smBGC, are likely to contribute to the environmental fitness of the strain [34-36]. Table 1 provides the links to data files 1-9.

We believe the dataset presented in Pseudomonas fragi strain A13BB chromosome, complete genome [39] and in this data note form a sound basis for further in-depth study of the metabolic and biosynthetic capabilities of this strain, and indeed of other closely related species. The dataset also provides useful insights into the molecular mechanisms that underpin these capabilities. 
Table 1 Overview of data files/data sets

\begin{tabular}{|c|c|c|c|}
\hline Label & Name of data file/data set & File types (file extension) & $\begin{array}{l}\text { Data repository and identifier (DOI or } \\
\text { accession number) }\end{array}$ \\
\hline $\begin{array}{l}\text { Data } \\
\text { file } 1\end{array}$ & Composition of ultra-minimal substrate growth medium & $\begin{array}{l}\text { Portable Document Format } \\
\text { file (.pdf) }\end{array}$ & $\begin{array}{l}\text { https://doi.org/10.6084/m9.figshare.12781193.v1 } \\
\text { [7] }\end{array}$ \\
\hline $\begin{array}{l}\text { Data } \\
\text { file } 2\end{array}$ & Quality distribution of Illumina reads & $\begin{array}{l}\text { Portable Network Graphic } \\
\text { file (.png) }\end{array}$ & $\begin{array}{l}\text { https://doi.org/10.6084/m9.figshare.13490967.v1 } \\
\text { [11] }\end{array}$ \\
\hline $\begin{array}{l}\text { Data } \\
\text { file } 3\end{array}$ & Basic quality statistics of GridION sequencing data & $\begin{array}{l}\text { Portable Document Format } \\
\text { file (.pdf) }\end{array}$ & $\begin{array}{l}\text { https://doi.org/10.6084/m9.figshare.13491147.v1 } \\
\text { [13] }\end{array}$ \\
\hline $\begin{array}{l}\text { Data } \\
\text { file } 4\end{array}$ & Average GridıON read quality plot & $\begin{array}{l}\text { Portable Network Graphic } \\
\text { file (.png) }\end{array}$ & $\begin{array}{l}\text { https://doi.org/10.6084/m9.figshare.13491210.v1 } \\
\text { [14] }\end{array}$ \\
\hline $\begin{array}{l}\text { Data } \\
\text { file } 5\end{array}$ & Quast report & $\begin{array}{l}\text { Portable Document Format } \\
\text { file (.pdf) }\end{array}$ & $\begin{array}{l}\text { https://doi.org/10.6084/m9.figshare.13491228.v1 } \\
\text { [17] }\end{array}$ \\
\hline $\begin{array}{l}\text { Data } \\
\text { file } 6\end{array}$ & Short BUSCO summary & $\begin{array}{l}\text { Portable Document Format } \\
\text { file (.pdf) }\end{array}$ & $\begin{array}{l}\text { https://doi.org/10.6084/m9.figshare.13491234.v1 } \\
\text { [19] }\end{array}$ \\
\hline $\begin{array}{l}\text { Data } \\
\text { file } 7\end{array}$ & Assembly graph & $\begin{array}{l}\text { Portable Network Graphic } \\
\text { file (.png) }\end{array}$ & $\begin{array}{l}\text { https://doi.org/10.6084/m9.figshare.14370608.v1 } \\
\text { [21] }\end{array}$ \\
\hline $\begin{array}{l}\text { Data } \\
\text { file } 8\end{array}$ & Predicted Genomic Islands of $P$. fragi A13BB & $\begin{array}{l}\text { Portable Document Format } \\
\text { file (.pdf) }\end{array}$ & $\begin{array}{l}\text { https://doi.org/10.6084/m9.figshare.13491300.v1 } \\
\text { [30] }\end{array}$ \\
\hline $\begin{array}{l}\text { Data } \\
\text { file } 9\end{array}$ & $\begin{array}{l}\text { Metabolic pathways of interest in P. fragi A13BB and } \\
\text { associated gene clusters }\end{array}$ & $\begin{array}{l}\text { Portable Document Format } \\
\text { file (.pdf) }\end{array}$ & $\begin{array}{l}\text { https://doi.org/10.6084/m9.figshare.13507971.v1 } \\
\text { [31] }\end{array}$ \\
\hline $\begin{array}{l}\text { Data } \\
\text { set } 1\end{array}$ & Illumina and GridION sequencing reads & Fastq file (.fastq.gz) & $\begin{array}{l}\text { https://identifiers.org/ncbi/insdc.sra:SRP251948 } \\
\text { [37] }\end{array}$ \\
\hline $\begin{array}{l}\text { Data } \\
\text { set } 2\end{array}$ & Genome assembly of $P$. fragi $\mathrm{A} 13 \mathrm{BB}$ & Fasta file (.fna) & $\begin{array}{l}\text { https://identifiers.org/insdc.gca:GCA_01576 } \\
7515.1 \text { [38] }\end{array}$ \\
\hline
\end{tabular}

Furthermore, being only the fourth publicly available complete genome sequence of $P$. fragi, the data will enrich the comparative genomics study of the species.

\section{Limitations}

IslandViewer 4 was run with default parameters. Crucially, IslandPick was run with default comparison genomes; different comparison genomes at different phyletic distances may influence the output of the analysis i.e. number of predicted genomic islands.

\section{Abbreviations \\ GC: Guanine-Cytosine; CDSs: Coding sequences; RNA: Ribonucleic acid; rRNA: Ribosomal ribonucleic acid; tRNA: Transfer ribonucleic acid; ncRNA: Non-coding ribonucleic acid; CRISPRs: Clustered regularly interspaced short palindromic repeats; PGPR: Plant growth-promoting rhizobacteria; smBGCs: Secondary metabolite biosynthetic gene clusters; \\ DNA: Deoxyribonucleic acid; gDNA: Genomic deoxyribonucleic acid; PCR: Polymerase chain reaction; ONT: Oxford nanopore technology; ANI: Average nucleotide identity; NAGGN: N-acetylglutaminylglutamine amide}

\section{Acknowledgements}

Illumina sequencing was performed by Glasgow Polyomics (http://www. glasgow.ac.uk/polyomics), GridION sequencing was provided by MicrobesNG (http://www.microbesng.uk). The authors would like to thank Dr. David McGuinness (Glasgow Polyomics) for the invaluable assistance with Illumina data analysis.

\section{Authors' contributions}

The project was conceived and designed by OKA and AJL. Data acquisition was performed by OKA. Data analysis and interpretation was performed by $\mathrm{OKA}, \mathrm{NHO}, \mathrm{ADS}$ and AJL. The project was jointly supervised by NHO, ADS and AJL. AJL was the principal investigator. The manuscript was written by
OKA and revised by NHO, ADS and AJL. All authors read and approved the final manuscript.

\section{Funding}

The project was supported by Tenovus Scotland (grant number G16.04). Tenovus Scotland played no role in the design of the study or the collection, analysis, and interpretation of data, or in writing the manuscript.

\section{Availability of data and materials}

Data files $1-9$ described in this Data note can be freely and openly accessed on Figshare (https://figshare.com/) [7, 11, 13, 14, 17, 19, 21, 30, 31]. Datasets 1 and 2 can be freely and openly accessed on the NCBI database. Illumina and GridION reads generated have been deposited in the Sequence Read Archive under accession number SRP251948 (Dataset 1) [37]. The genome assembly of $P$. fragi A13BB has been deposited in GenBank under accession number GCA_015767515.1 (Dataset 2) [38]. The BioProject accession number for the entire project is PRJNA610978. Please see Table 1 and references for details and links to the data.

\section{Declarations}

Ethics approval and consent to participate

Soil sampling was undertaken on private land in Aberdeen, Scotland, UK with full landowner permission.

\section{Consent for publication}

Not applicable.

\section{Competing interests}

The authors declare no competing interests.

\section{Author details}

'School of Pharmacy and Life Sciences, Robert Gordon University, Sir lan Wood Building, Garthdee Road, Aberdeen AB10 7GJ, Scotland. ${ }^{2}$ Graduate School, Robert Gordon University, The Ishbel Gordon Building, Garthdee Road, Aberdeen AB10 7QE, Scotland. 
Received: 19 January 2021 Accepted: 4 May 2021

Published online: 18 May 2021

\section{References}

1. Raaijmakers JM, Paulitz TC, Steinberg C, Alabouvette C, Moënne-Loccoz Y. The rhizosphere: a playground and battlefield for soilborne pathogens and beneficial microorganisms. Plant Soil. 2009;321(1-2):341-61. https://doi.org/1 0.1007/s11104-008-9568-6.

2. Lugtenberg B, Kamilova F. Plant-growth-promoting rhizobacteria. Annu Rev Microbiol. 2009;63(1):541-56. https://doi.org/10.1146/annurev.micro.62.0813 07.162918 .

3. Bérdy J. Thoughts and facts about antibiotics: where we are now and where we are heading. J Antibiot. 2012;65(8):385-95. https://doi.org/10.103 8/ja.2012.27.

4. Ercolini D, Casaburi A, Nasi A, Ferrocino I, Monaco RD, Ferranti P, et al. Different molecular types of Pseudomonas fragi have the same overall behaviour as meat spoilers. Int J Food Microbiol. 2010;142(1-2):120-31. https://doi.org/10.1016/j.ijfoodmicro.2010.06.012.

5. Selvakumar G, Joshi P, Nazim S, Mishra P, Bisht J, Gupta H. Phosphate solubilization and growth promotion by Pseudomonas fragi CS11RH1 (MTCC 8984), a psychrotolerant bacterium isolated from a high altitude Himalayan rhizosphere. Biologia. 2009;64(2):239-45. https://doi.org/10.2478/s11756-0090041-7.

6. Farh ME, Kim YJ, Sukweenadhi J, Singh P, Yang DC. Aluminium resistant, plant growth promoting bacteria induce overexpression of aluminium stress related genes in Arabidopsis thaliana and increase the ginseng tolerance against aluminium stress. Microbiol Res. 2017;200:45-52. https://doi.org/10.1 016/j.micres.2017.04.004.

7. Data File 1: Composition of ultra-minimal substrate growth medium. Figshare. https://doi.org/10.6084/m9.figshare.12781193.v1 (2020).

8. Bolger AM, Lohse M, Usadel B. Trimmomatic: a flexible trimmer for Illumina sequence data. Bioinformatics. 2014;30(15):2114-20. https://doi.org/10.1093/ bioinformatics/btu170.

9. Andrews S. FastQC: a quality control tool for high throughput sequence data. http://www.bioinformatics.babraham.ac.uk/projects/fastac/ (2010).

10. Ewels P, Magnusson M, Lundin S, Käller M. MultiQC: summarize analysis results for multiple tools and samples in a single report. Bioinformatics. 2016;32(19):3047-8. https://doi.org/10.1093/bioinformatics/btw354.

11. Data file 2: Quality distribution of Illumina reads. Figshare. https://doi.org/10 6084/m9.figshare.13490967.v1 (2020).

12. De Coster W, D'Hert S, Schultz DT, Cruts M, Van Broeckhoven C. NanoPack: visualizing and processing long-read sequencing data. Bioinformatics. 2018; 34(15):2666-9. https://doi.org/10.1093/bioinformatics/bty149.

13. Data file 3: Basic quality statistics of GridION sequencing data. Figshare. https://doi.org/10.6084/m9.figshare.13491147.v1 (2020).

14. Data File 4: Average GridlON read quality plot. Figshare. https://doi.org/10. 6084/m9.figshare.13491210.v1 (2020).

15. Wick RR, Judd LM, Gorrie CL, Holt KE. Unicycler: resolving bacterial genome assemblies from short and long sequencing reads. PLoS Comput Biol. 2017; 13(6):1-22. https://doi.org/10.1371/journal.pcbi.1005595.

16. Gurevich A, Saveliev V, Vyahhi N, Tesler G. QUAST: quality assessment tool for genome assemblies. Bioinformatics. 2013;29(8):1072-5. https://doi.org/1 0.1093/bioinformatics/btt086.

17. Data file 5: Quast report. Figshare. https://doi.org/10.6084/m9.figshare.134 91228.v1 (2020).

18. Simão FA, Waterhouse RM, loannidis $P$, Kriventseva EV, Zdobnov EM BUSCO: assessing genome assembly and annotation completeness with single-copy orthologs. Bioinformatics. 2015;31(19):3210-2. https://doi.org/1 0.1093/bioinformatics/btv351.

19. Data file 6: Short BUSCO summary. Figshare. https://doi.org/10.6084/m9. figshare.13491234.v1 (2020).

20. Wick RR, Schultz MB, Zobel J, Holt KE. Bandage: interactive visualization of de novo genome assemblies. Bioinformatics. 2015;31 (20):3350-2. https://doi. org/10.1093/bioinformatics/btv383.

21. Data file 7: Assembly graph. Figshare. https://doi.org/10.6084/m9.figsha re.14370608.v1 (2021).

22. Jain C, Rodriguez-R LM, Phillippy AM, Konstantinidis KT, Aluru S. High throughput ANI analysis of 90K prokaryotic genomes reveals clear species boundaries. Nat Commun. 2018;9(1):5114. https://doi.org/10.1038/s41467-01 8-07641-9.
23. Tatusova T, DiCuccio M, Badretdin A, Chetvernin V, Nawrocki EP, Zaslavsky L, et al. NCBI prokaryotic genome annotation pipeline. Nucleic Acids Res. 2016; 44(14):6614-24. https://doi.org/10.1093/nar/gkw569.

24. Brettin T, Davis JJ, Disz T, Edwards RA, Gerdes S, Olsen GJ, et al. RASTtk: a modular and extensible implementation of the RAST algorithm for building custom annotation pipelines and annotating batches of genomes. Sci Rep. 2015;5(1):8365. https://doi.org/10.1038/srep08365.

25. Kanehisa M, Goto S. KEGG: Kyoto encyclopedia of genes and genomes. Nucleic Acids Res. 2000;28(1):27-30. https://doi.org/10.1093/nar/28.1.27.

26. Couvin D, Bernheim A, Toffano-Nioche C, Touchon M, Michalik J, Néron B, et al. CRISPRCasFinder, an update of CRISRFinder, includes a portable version, enhanced performance and integrates search for Cas proteins. Nucleic Acids Res. 2018;46(W1):W246-51. https://doi.org/10.1093/nar/gky425.

27. Bertelli C, Laird MR, Williams KP, Simon Fraser University Research Computing Group, Lau BY, et al. IslandViewer 4: expanded prediction of genomic islands for larger-scale datasets. Nucleic Acids Res. 2017;45:W30-5. https://doi.org/10.1093/nar/gkx343.

28. Arndt D, Grant JR, Marcu A, Sajed T, Pon A, Liang Y, et al. PHASTER: a better, faster version of the PHAST phage search tool. Nucleic Acids Res. 2016; 44(W1):W16-21. https://doi.org/10.1093/nar/gkw387.

29. Blin K, Shaw S, Steinke K, Villebro R, Ziemert N, Lee SY, et al. AntiSMASH 5.0: updates to the secondary metabolite genome mining pipeline. Nucleic Acids Res. 2019;47(W1):W81-7. https://doi.org/10.1093/nar/gkz310.

30. Data file 8: Predicted Genomic Islands of P. fragi A13BB. Figshare. https://doi. org/10.6084/m9.figshare.13491300.v1 (2020).

31. Data file 9: Metabolic pathways of interest in P. fragi A13BB and associated gene clusters. Figshare. https://doi.org/10.6084/m9.figshare.13507971.v1 (2020).

32. Robinson SL, Christenson JK, Wackett LP. Biosynthesis and chemical diversity of $\beta$-lactone natural products. Nat Prod Rep. 2019;36(3):458-75. https://doi. org/10.1039/c8np00052b.

33. Champomier-Vergès MC, Stintzi A, Meyer JM. Acquisition of iron by the non-siderophore-producing Pseudomonas fragi. Microbiology. 1996;142(5): 1191-9. https://doi.org/10.1099/13500872-142-5-1191.

34. Schöner TA, Gassel S, Osawa A, Tobias NJ, Okuno Y, Sakakibara Y, et al. Aryl Polyenes, a highly abundant class of bacterial natural products, are functionally related to Antioxidative carotenoids. Chembiochem. 2016;17(3): 247-53. https://doi.org/10.1002/cbic.201500474.

35. Sagot B, Gaysinski M, Mehiri M, Guigonis JM, Le Rudulier D, et al. Osmotically induced synthesis of the dipeptide $\mathrm{N}$-acetylglutaminylglutamine amide is mediated by a new pathway conserved among bacteria. Proc Natl Acad Sci U S A. 2010;107(28):12652-7. https://doi.org/10.1073/pnas.1003 063107.

36. Saha M, Sarkar S, Sarkar B, Sharma BK, Bhattacharjee S, Tribedi P. Microbial siderophores and their potential applications: a review. Environ Sci Pollut Res Int. 2016;23(5):3984-99. https://doi.org/10.1007/s11356-015-4294-0.

37. National Center for Biotechnology Information. Sequence Read Archive. https://identifiers.org/ncbi/insdc.sra:SRP251948 (2020).

38. National Center for Biotechnology Information. Assembly. https://identifiers. org/insdc.gca:GCA_015767515.1 (2020).

39. Awolope OK, Di Salvo A, O'Driscoll NH, Lamb AJ. Pseudomonas fragi strain A13BB chromosome, complete genome. GenBank. https://identifiers.org/ insdc:CP065202. 2020.

\section{Publisher's Note}

Springer Nature remains neutral with regard to jurisdictional claims in published maps and institutional affiliations.

\section{Ready to submit your research? Choose BMC and benefit from}

- fast, convenient online submission

- thorough peer review by experienced researchers in your field

- rapid publication on acceptance

- support for research data, including large and complex data types

- gold Open Access which fosters wider collaboration and increased citations

- maximum visibility for your research: over $100 \mathrm{M}$ website views per year

At $\mathrm{BMC}$, research is always in progress.

Learn more biomedcentral.com/submission 